\title{
On the reconstruction of diffusions from first-exit time distributions
}

\author{
Guillaume $\mathrm{Bal}^{1}$ and Tom $\mathrm{Chou}^{2}$ \\ ${ }^{1}$ Department of Applied Physics and Applied Mathematics, Columbia \\ University, New York NY, 10027, USA; gb2030@columbia.edu \\ ${ }^{2}$ Department of Biomathematics, UCLA, Los Angeles, CA, 90095, USA; \\ tomchou@ucla.edu
}

\begin{abstract}
.
This paper explores the reconstruction of drift or diffusion coefficients of a scalar stochastic diffusion processes as it starts from an initial value and reaches, for the first time, a threshold value. We show that the distribution function derived from repeated measurements of the first-exit times can be used to formally partially reconstruct the dynamics of the process. Upon mapping the relevant stochastic differential equations (SDE) to the associated Sturm-Liouville problem, results from Gelfand and Levitan [10] can be used to reconstruct the potential of the Schrödinger equation, which is related to the drift and diffusion functionals of the SDE. We show that either the drift or the diffusion term of the stochastic equation can be uniquely reconstructed, but only if both the drift and diffusion are known in at least half of the domain. No other information can be uniquely reconstructed unless additional measurements are provided. Applications and implementations of our results are discussed.
\end{abstract}

\section{Introduction}

Consider a diffusive stochastic process $X_{t}$, with an initial condition $X_{0}$. Once $X_{t}$ reaches a certain threshold value $X^{*}$, the process is stopped and restarted at $X_{0}$. The distribution of times for $X_{t}$ to first reach $X^{*}$ across the ensemble of measurements, the first-exit time distribution, arises in many physical applications 9, 24. Mean firstexit times are readily calculated from explicit forms for the dynamical rules controlling the process $X_{t}$. However, in many applications, the underlying physics is unknown, complex, and/or very difficult to model. In such instances, the question arises as to whether one can reconstruct the form of the governing equations or equation parameters that best fit measured data (such as first-exit time distributions). In this paper, we analyse a general scalar diffusive process to formally determine how much of the drift and diffusion functions can be determined from first-exit time data.

Many first passage time problems can be recast as inverse problems. We describe two physical realizations that motivate this work: transmembrane voltage spike frequencies in neurons [11, 31, and first passage times over a molecular barrier in the rupture of chemical bonds [9] 16. The transmembrane voltage of a nerve cell is routinely measured and typically exhibits repeated, sharp spikes. A class of reduced models for the transmembrane voltage $V$ is described by

$$
\frac{d V_{t}}{d t}=I\left(V_{t}\right)+g\left(V_{t}\right) \eta_{t}
$$


where $I\left(V_{t}\right)$ and $g\left(V_{t}\right)$ are typically polynomial functions of $V$, and $\eta_{t}$ is a noise arising from interactions with the many other connected neurons. This Langevintype equation is to be appropriately interpreted with Stratonovich calculus [15]. An additional, instantaneous nonlinearity that gives rise to a spike and voltage reset is implicitly included by imposing a threshold voltage $V^{*}$. This nonlinearity arises ultimately from the nonlinear dynamics of ion channels that span the cell membrane [11. Upon reaching $V^{*}$, the system instantaneously spikes and resets to the value $V_{0}$. Various forms of $I\left(V_{t}\right)$ and $g\left(V_{t}\right)$ have found wide use. For example, when the noise $\eta_{t}$ arising from other connected nerve cells changes the nerve cell's membrane conductivity, $g\left(V_{t}\right) \propto V_{t}+$ const. Both linear (linear integrate-and-fire) and quadratic (quadratic integrate-and-fire) forms are used to model $I\left(V_{t}\right)$ [11] 21, although it is often unclear which model of $I\left(V_{t}\right)$ is most appropriate [21]. Instead of solving for the spike times for presupposed $I\left(V_{t}\right)$ and $g\left(V_{t}\right)$ [30, our goal is to infer as much as possible about the nonlinear current-voltage relationship $I\left(V_{t}\right)$ and coupling to noisy inputs $g\left(V_{t}\right)$, given a measured spike time distribution [29].

Another application where first exit times can be used to reconstruct models is in the single molecule measurements of macromolecular detachment, or bond rupturing. An example of this rapidly developing field is the accelerated rupturing of bonds upon application of a dynamic load between two macromolecules, such as the ligandreceptor complex biotin-streptavidin [20, 27]. The mean times to bond breaking have been measured [20] and modelled [27]. This "Kramers problem" [9, 16, 24], can also be studied as an inverse problem. Bond rupture can be modelled as the irreversible passage of the relevant molecular coordinate $X$ over a barrier from a metastable state. Thermal bombardments agitate both the molecular distance coordinate directly and the interaction potential. In the overdamped, or Markovian limit, the distance coordinate obeys the Langevin equation

$$
\frac{d X_{t}}{d t}=-U^{\prime}\left(X_{t}\right)+g\left(X_{t}\right) \eta_{t},
$$

where $U\left(X_{t}\right)$ is the effective interaction potential of the rupturing bond, and $g\left(X_{t}\right)$ represents a force that arises from a fluctuating potential driven by the white diffusion noise $\eta_{t}$ (again, the appropriate convention is the Stratonovich difference rule). The forward problem of bond rupture, or detachment, using simple fixed potentials $U\left(X_{t}\right)$ 9] 16, and with explicit forms for fluctuating potentials [7, have been well-studied.

Both problems discussed above (see Fig. 1), as well as many others, can be modeled using a scalar stochastic process where the first-exit time distribution obeys the backward Kolmogorov equation. We show in the following sections, that the measured first-exit time distribution (given a fixed initial condition) along with knowledge of the drift and diffusion parameters in part of the domain, is sufficient to uniquely determine one of them in the entire domain provided the other one is known. Our analysis gives a formal procedure for reconstructing drift or diffusion of the stochastic process. It is based on transforming the equation into a Schrödinger type equation and on analytical (more precisely meromorphic) continuation of measured data to obtain the spectral data of the Schrödinger equation. Although uniqueness is demonstrated in the aforementioned framework, the necessary analytical continuation in the reconstruction shows that the inverse problem is severely ill-posed [8]. 


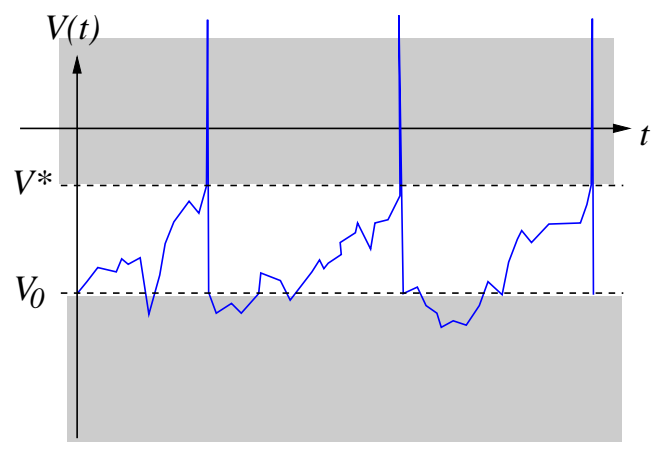

(a)

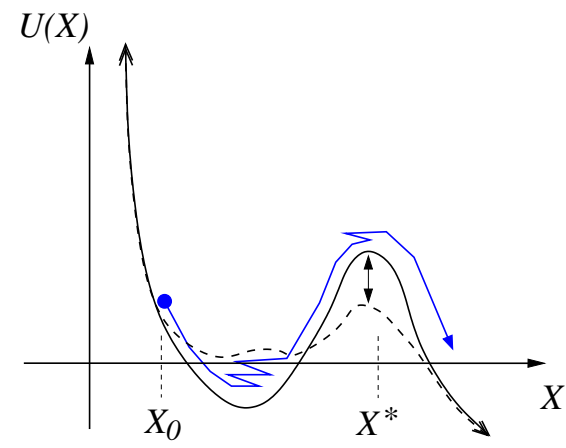

(b)

Figure 1. (a) The transmembrane potential is instantaneously reset to $V_{0}$ as soon as it crosses $V^{*}$. We demonstrate that the functional form of the voltage dynamics can be be determined only between $V_{0}$ and $V^{*}$ (the unshaded region). (b) Passage over a metastable barrier. The first-passage, bond-breaking times can be used to construct the potential $U(X)$ and and how it fluctuates (dashed curve).

\section{Stochastic Equations and Main Result}

Consider the general stochastic process

$$
d X_{t}=\mu\left(X_{t}\right) d t+\sigma\left(X_{t}\right) d W_{t},
$$

where $d W_{t}=\eta_{t} d t$ is the Wiener measure, and the Itô interpretation is used [15, 22]. The drift term, $\mu(X)$, is assumed to have sub-linear growth at infinity, and is of class $C^{1}(\mathbb{R})$. We also assume that the variance, $\sigma(X)$, is uniformly bounded from above and below by positive constants, and is of class $C^{2}(\mathbb{R})$. These assumptions are sufficient to ensure that the process (3) admits a unique solution [22].

In the application to spiking action potentials in neurons, $X_{t} \equiv V_{t}$ is the transmembrane voltage at time $t$. The potential immediately after reset is denoted $X_{0} \equiv V_{0}$. The corresponding drift and diffusion terms are defined as $\mu(X) \equiv$ $I(X)+\frac{1}{2} g(X) g^{\prime}(X)$ and $\sigma(X) \equiv g(X)$, respectively, since (3) is in the Itô form and (11) should be interpreted in the Stratonovich sense [22]. For a more detailed derivation of the the diffusion process in the context of action potential modeling, we refer the reader to number of standard works [11, 14, 17, 31. In the application to chemical bond rupturing, $X$ denotes the reaction coordinate along an effective molecular energy landscape (cf. Fig. 1(b)), $X_{0}$ is an initial bond displacement, and we identify the drift in (3) with $-U^{\prime}(X)+\frac{1}{2} g(X) g^{\prime}(X)$.

Let us now consider the interval $\Delta=\left(X_{-}, X^{*}\right)$ and the first exit time $t^{*}$ of the process $X_{t}$; i.e., $t^{*}$ is the first time of exit of $X_{t}$ out of $\left(X_{-}, X^{*}\right)$ [4] 17] 22]. First exit time measurements can then be used to reconstruct properties of the diffusion process. For instance, it is known that knowledge of $P_{X}\left(X_{t^{*}}=X^{*}\right)$ (the probability that $X_{t}$ exits $\Delta$ at $X^{*}$ conditional on it starting at $X \in \Delta$ ) and $\mathbf{E}_{X}\left[t^{*}\right]$ (the expected time it takes for $X_{t}$ to exit $\Delta$ starting at $X$ ), uniquely determines $\mu$ and $\sigma$. We refer to Breiman [4 for details on the so-called natural time scale and speed measure of diffusion processes. 
In the applications presented here, the situation is different: We have access to the full distribution of the first-exit times, but only for processes starting at $X=X_{0}$ (the reset potential or the initial relative molecular coordinate). The distribution of the exit times can be conveniently written as the solution to a partial differential equation (the backward Kolmogorov equation) 9, 12, 17, 24, 28, 31. Let us denote by $w(X ; \lambda)$ the Laplace transform (from the time variable $t$ to the dual variable $\lambda$ ) of the probability density $\tilde{w}(X ; t)$ of the first-exit times $t$ from $X \in \Delta$ of the diffusion process starting at $X=X_{0}$. For simplicity, we will assume reflecting boundary conditions for the diffusion at $X=X_{-}$, and consider termination of the process only at $X^{*}$. Following [9, 12, 17, 28, we find that $w(X ; \lambda)$ solves the following backward Kolmogorov equation,

$$
\begin{gathered}
\frac{\sigma^{2}(X)}{2} w^{\prime \prime}(X ; \lambda)+\mu(X) w^{\prime}(X ; \lambda)=\lambda w(X ; \lambda), \quad X_{-}<X<X^{*}, \quad \lambda>0, \\
w\left(X^{*} ; \lambda\right)=1, \quad \frac{\partial w\left(X_{-} ; \lambda\right)}{\partial X}=0,
\end{gathered}
$$

where $\partial w\left(X_{-} ; \lambda\right) / \partial X=0$ corresponds to vanishing probability flux at $X=X_{-}$. Although we restrict our analysis to a reflecting lower bound, depending on the physical application, other boundary conditions can be imposed at an arbitrary lower bound. The boundary condition $w\left(X^{*} ; \lambda\right)=1$ reflects the fact that $\tilde{w}\left(X_{0}=X^{*}, t\right)=$ $\delta(t)$. Since $\tilde{w}\left(X_{0} ; t\right)$ is directly measured, $w\left(X_{0} ; \lambda\right)$ is exactly determined for all $\lambda>0$. Using an affine change of variables, $\left(X-X_{-}\right) /\left(X^{*}-X_{-}\right) \rightarrow x$, we rescale the domain to $x \in[0,1]$ and recast Eq. (4) as

$$
\begin{aligned}
& \frac{\sigma^{2}(x)}{2} w^{\prime \prime}(x ; \lambda)+\mu(x) w^{\prime}(x ; \lambda)=\lambda w(x ; \lambda), \quad 0<x<1, \quad \lambda>0, \\
& w(1 ; \lambda)=1, \quad \frac{\partial w(0 ; \lambda)}{\partial x}=0,
\end{aligned}
$$

where $x$ is now the initial position of the process. We still use the notation $\sigma$ and $\mu$ for the diffusion and drift terms in the rescaled variables. For example, in the neuron spiking problem, the voltage threshold is at 1 , the inhibitory threshold (the lower bound for the voltage) is at 0 , and the reset voltage that restarts the process is at $x=x_{0}$. This problem admits a unique solution provided $\sigma$ remains uniformly bounded from above and below and $\mu$ remains bounded.

In many instances $\mu(x)$ and $\sigma(x)$ are known from a well-accepted model, and the function $w(x ; \lambda)$ can be computed directly. Now consider the case where $\mu(x)$ and $\sigma(x)$ are unknown, but $w\left(x=x_{0} ; \lambda\right)$ is measured at a single initial position $x_{0}=\left(X_{0}-X_{-}\right) /\left(X^{*}-X_{-}\right)$. What can one reconstruct (with respect to $\mu(x)$ and $\sigma(x))$ from a knowledge of $w\left(x_{0} ; \lambda\right)$ for all $\lambda>0$ ? In answering this question, we arrive at the following:

Let $0<x_{b}<1$ be the point defined in (18) below and let $\Lambda \subset(0,1)$ be a countable set of Lebesgue measure 0 in $(0,1)$, defined in (23) below. We obtained the following related results:

Theorem 1 Assume that $\mu(x)$ and $\sigma(x)$ are known on $\left(0, x_{b}\right)$ and that the measurement point $x_{0} \in(0,1) \backslash \Lambda$. If, in addition, either $\mu(x), \sigma(x)$, or $\mu(x) / \sigma(x)$ is known entirely on $[0,1]$, then both $\mu(x)$ and $\sigma(x)$ are uniquely determined on $[0,1]$ by measurement of $w\left(x_{0} ; \lambda\right)$, for all $\lambda>0$.

Theorem 2 Assume that $\mu(x)$ and $\sigma(x)$ are known on $\left(0, x_{0}\right)$ and that the measurement point $x_{0} \geq x_{b}$. If, in addition, either $\mu(x), \sigma(x)$, or $\mu(x) / \sigma(x)$ is known 
on $[0,1]$, then both $\mu(x)$ and $\sigma(x)$ are uniquely determined on $[0,1]$ by measurement of $w\left(x_{0} ; \lambda\right)$, for all $\lambda>0$.

Both theorems say that if either $\mu$ or $\sigma$ is known on $[0,1]$, while the other is known only on half of the domain, we can reconstruct this other function also on the entire domain. Since $x_{b}$ is implicitly defined by $\sigma$ (cf. Eq. 18), it is an a priori constraint on the size of the domain that one can reconstruct from the measurements $w\left(x_{0} ; \lambda\right)$. In Theorem 1 , the measurements are made at an almost arbitrary point $x_{0} \in(0,1)$. We have to remove a countable set of points, which is also defined implicitly as it depends on $\sigma$ and $\mu$. This constraint is no longer necessary in Theorem 2, when $x_{0} \in\left(x_{b}, 1\right)$.

The conclusions of both results hold true if the domains $\left(0, x_{b}\right)$ and $\left(0, x_{0}\right)$ of $a$ priori knowledge of the coefficients are replaced by $\left(x_{b}, 1\right)$ and $\left(x_{0}, 1\right)$, respectively with $x_{0} \leq x_{b}$ in the latter case. We do not explicitly consider these cases. The main steps of the proof are the following. First we use a transformation similar to the Liouville transformation (used to obtain the canonical form of Sturm-Liouville problems [6]) to map Eq. (5) into a Schrödinger equation (Section 3). Next, we show that a measurement of $w\left(x_{0} ; \lambda\right)$, for all $\lambda>0$, is sufficient to obtain a single eigenvalue spectrum of the Sturm-Liouville problem (when $x_{0} \notin \Lambda$ in the setting of Theorem 1). This allows us to use results from Gelfand and Levitan [10] to verify uniqueness of the reconstruction (Section 4) and explicitly extract $\mu(x)$ or $\sigma(x)$ (Section 5 ). The above theorems require to know the coefficients on half of the domain before one of them can be reconstructed on the other half. An explicit asymptotic expansion presented in section 5 shows that this a priori knowledge is indeed necessary to obtain a unique reconstruction. In section 6 we present a result showing that the reconstruction of one of the coefficients $\mu(x)$ or $\sigma(x)$ is unique over the whole domain $(0,1)$ provided that they are known on an arbitrarily small domain $(0, \eta)$ and provided that a sequence of measurements $w\left(x_{m} ; \lambda\right)$ is available for $\lambda>0$ and $x_{m}, m \in \mathbb{N}$, judiciously chosen. In this result, a minimum of $M$ measurements is required to reconstruct $\mu$ or $\sigma$ provided that they are known a priori on an interval close to $x=0$ of size $2^{-M}$. Finally section 7 considers possible implementations of the method.

\section{Mapping to Schrödinger form}

To recast Eq. (5) as a Schrödinger equation, we effect the following change of variables

$$
w(x)=(f u) \circ h(x) .
$$

Thus,

$$
w^{\prime}(x) \Rightarrow h^{\prime}(f u)^{\prime} \circ h, \quad w^{\prime \prime}(x) \Rightarrow h^{\prime \prime}(f u)^{\prime} \circ h+\left(h^{\prime}\right)^{2}(f u)^{\prime \prime} \circ h,
$$

and Eq. (5) becomes

$$
\left[\frac{\sigma^{2}}{2}\left(h^{\prime}\right)^{2}\right](f u)^{\prime \prime} \circ h+\left[\frac{\sigma^{2}}{2} h^{\prime \prime}+\mu h^{\prime}\right](f u)^{\prime} \circ h=\lambda(f u) \circ h .
$$

To normalise the second-order term, we impose the constraint

$$
\begin{aligned}
& h^{\prime}(x)=\frac{\sqrt{2}}{\sigma(x)}, \quad 0<x<1, \\
& h(0)=0 .
\end{aligned}
$$


Since $\sigma$ is bounded from above and below by a positive constant, $x \rightarrow h(x)$ is a diffeomorphism. Upon introducing the change of variables

$$
y=h(x),
$$

the points $x=0, x=x_{0}$, and $x=x_{b}$ before the change of variables become $y=h(0)=0, y_{0} \equiv h\left(x_{0}\right)$, and $y_{b} \equiv h\left(x_{b}\right)$, respectively. If we define a new drift

$$
\nu(y)=\left(\frac{\sigma^{2}}{2} h^{\prime \prime}+\mu h^{\prime}\right)\left(h^{-1}(y)\right), \quad 0 \leq y \leq y^{*} \equiv h(1),
$$

Eq. (8) can be succinctly expressed as

$$
(f u)^{\prime \prime}(y)+\nu(y)(f u)^{\prime}(y)=\lambda(f u)(y) .
$$

To remove the drift term, we force $f$ to be the solution to

$$
\begin{aligned}
& 2 f^{\prime}(y)+\nu(y) f(y)=0, \quad 0<y<y^{*}, \\
& f\left(y_{0}\right)=1 .
\end{aligned}
$$

Since $f(y)>0$ for $y=y_{0}, f(y)>0$ for all $y \in\left[0, y^{*}\right]$. We finally obtain

$$
\begin{aligned}
& u^{\prime \prime}(y)-q(y) u(y)=\lambda u(y), \quad 0<y<y^{*} \\
& u\left(y^{*}\right)=\frac{1}{f\left(y^{*}\right)}>0, \quad \frac{\partial u(0 ; \lambda)}{\partial y}=0,
\end{aligned}
$$

where the potential is defined by

$$
q(y)=-\frac{\nu f^{\prime}+f^{\prime \prime}}{f}(y) .
$$

By assumption, $\sigma(x)$ and $\mu(x)$ are known for $0 \leq x \leq b$. This implies that $\nu(y)$ and $f(y)$, and hence $q(y)$, are known for $0 \leq y \leq y_{b} \equiv h\left(x_{b}\right)$, although $y^{*} \equiv h(1)$ itself is generally not known (unless $\sigma(x)$ is also known on $[0,1]$ ). This undetermined domain size is found in the following section.

\section{Uniqueness of the potential reconstruction}

We now consider the reconstruction of the potential $q(y)$ in the equation

$$
\begin{aligned}
& u^{\prime \prime}(y ; \lambda)-q(y) u(y ; \lambda)=\lambda u(y ; \lambda), \quad 0<y<y^{*} \equiv h(1) \\
& u\left(y^{*}\right)=\frac{1}{f\left(y^{*}\right)}>0, \quad \frac{\partial u(0 ; \lambda)}{\partial y}=0 .
\end{aligned}
$$

The assumption is that $q(y)$ is known on $\left[0, y_{b}\right]$ as described earlier. However $y^{*}=h(1)$ depends on $\sigma(x)$ throughout the domain and is unknown, as is $q(y)$ on $\left(0, y^{*}\right)$. From measurements, we have $u\left(y_{0} ; \lambda\right)$ for all $\lambda>0$ since $w\left(x_{0} ; \lambda\right)=u\left(y_{0} ; \lambda\right)$ and $f\left(y_{0}\right)=1$.

Uniqueness of the reconstruction of $q(y)$ on $\left(y_{b}, y^{*}\right)$ is based on the fact that $\lambda \rightarrow u(y ; \lambda)$ is analytic with poles corresponding to values of $\lambda$ that are eigenvalues for specific spectral equations. It is known that two spectral equations are necessary to reconstruct the potential $q(y)$ from spectral data [3, 6, 10, 18]. However, we will see that our problem allows extraction of at most only one spectrum. Nonetheless, if the "potential" $q(y)$ is known for half of the entire domain, one eigenvalue spectrum suffices for the complete and unique reconstruction of $q(y)$ [6, 26]. 
Since $q(y)$ is known for $0 \leq y \leq h\left(x_{b}\right)$, we require

$$
h\left(x_{b}\right)=\frac{y^{*}}{2} \equiv \frac{h(1)}{2}
$$

in order for $q(y)$ to be completely reconstructed on $\left[0, y^{*}\right]$ Therefore, the original functions $\mu(x)$ and $\sigma(x)$ must at least be known for $0 \leq x \leq x_{b}$, where

$$
x_{b}=h^{-1}\left(\frac{y^{*}}{2}\right)=h^{-1}\left(\frac{h(1)}{2}\right) .
$$

The full problem on $\left[0, y^{*}\right]$ becomes

$$
\begin{aligned}
& u^{\prime \prime}(y)-q(y) u(y)=\lambda u(y), \quad 0<y<y^{*} \equiv h(1) \\
& u\left(y^{*}\right)=\frac{1}{f\left(y^{*}\right)} \neq 0, \quad \frac{\partial u(0 ; \lambda)}{\partial y}=0 . \\
& u\left(y_{0} ; \lambda\right), \quad \text { known. }
\end{aligned}
$$

Now, consider the associated eigenvalue problem

$$
\begin{aligned}
& \phi_{k}^{\prime \prime}(y)-q(y) \phi_{k}(y)=-\lambda_{k} \phi_{k}(y), \quad 0<y<y^{*}, \quad k \geq 1 \\
& \phi_{k}^{\prime}(0)=\phi_{k}\left(y^{*}\right)=0 .
\end{aligned}
$$

If the $\lambda_{k}$ in (20) can be determined from our measured data $u\left(y_{0} ; \lambda\right)$, then $q(y)$ on $\left[0, y^{*}\right]$ can be determined upon using classic theories described in [3, 6, 10, 13, 18, 25]. To find the eigenvalues to (20), as well as the transformed threshold $y^{*}$, we decompose the Green's function for (19) in terms of the eigenfunctions of (20), and obtain

$$
u(y ; \lambda)=-\sum_{k}\left[\frac{\phi_{k}(y) \phi_{k}^{\prime}\left(y^{*}\right) u\left(y^{*}\right)}{\lambda+\lambda_{k}}\right] .
$$

First, consider the setting of Theorem 1. Since $u\left(y^{*}\right)=1 / f\left(y^{*}\right)>0$ and $\left|\phi_{k}^{\prime}\left(y^{*}\right)\right|>0$ (since $\phi_{k}$ is a non-trivial solution to (20)), $\left|u\left(y_{0} ; \lambda\right)\right| \rightarrow \infty$ as $\lambda \rightarrow-\lambda_{k}$, provided $\phi_{k}\left(y_{0}\right) \neq 0$. The latter constraint is not satisfied for all choices of $y_{0}$. Let us define

$$
\Lambda_{y}=\left\{y \in\left(0, y^{*}\right), \text { there exists } k \geq 1 \text { such that } \phi_{k}(y)=0\right\} .
$$

Since the number of zeros of solutions to the Sturm-Liouville problem (20) is finite, the set $\Lambda_{y}$ is countable and therefore of Lebesgue measure 0 on $\left(0, y^{*}\right)$. As long as our initial measurement position $y_{0}$ is not part of the above countable subset of values where the eigenfunctions of (20) have a node, we can in principle obtain one spectrum by analytically continuing the data $u\left(y_{0} ; \lambda\right)$ in $\lambda$ and finding the poles. The constraint on $y_{0}$ also amounts to saying that the eigenfunctions $\phi_{k}$ cannot be restricted to being eigenvectors of the same problem posed on the domain $\left(0, y_{0}\right)$ instead of $\left(0, y^{*}\right)$. In the primitive variables the countable set of forbidden measurement points is thus defined as

$$
\Lambda=h^{-1}\left(\Lambda_{y}\right)=\left\{x \in(0,1), h(x) \in \Lambda_{y}\right\} .
$$

Now consider Theorem 2. We can extend $u(y ; \lambda)$ by evenness on $\left(-y^{*}, y^{*}\right)$ and then by periodicity on $\mathbb{R}$. The resulting function is then continuous in $y$ and we deduce from (21) that

$$
\frac{\partial u}{\partial y}(y ; \lambda)=-\sum_{k}\left[\frac{\phi_{k}^{\prime}(y) \phi_{k}^{\prime}\left(y^{*}\right) u\left(y^{*}\right)}{\lambda+\lambda_{k}}\right] .
$$


Now since $\mu(x)$ and $\sigma(x)$ are known on $\left(0, x_{0}\right)$ then so is $q(y)$ on $\left(0, y_{0}\right)$. Since $u\left(y_{0}, \lambda\right)$ is known, we can solve (14) for $u(y)$ on $\left(0, y_{0}\right)$ and thus obtain the value of $\frac{\partial u}{\partial y}\left(y_{0} ; \lambda\right)$. Since either $\left|\phi_{k}\left(y_{0}\right)\right|>0$ or $\left|\phi_{k}^{\prime}\left(y_{0}\right)\right|>0$ (for otherwise $\phi_{k}(y) \equiv 0$ ), we deduce that $\left|u\left(y_{0} ; \lambda\right)\right|+\left|\frac{\partial u}{\partial y}\left(y_{0} ; \lambda\right)\right| \rightarrow \infty$ as $\lambda \rightarrow-\lambda_{k}$. Under the hypotheses of Theorem 1 and Theorem 2, we thus find that one can reconstruct the spectrum $\left\{\lambda_{k}\right\}_{k \geq 1}$ in (20) from the measurements.

Finally, we must determine $y^{*}$. Asymptotic expansions of the eigenvalues for large $k$, found using the WKB approximation 22, 6, 25] have the form:

$$
\lambda_{k}=\left[\left(k-\frac{1}{2}\right) \frac{\pi}{y^{*}}\right]^{2}+\frac{1}{y^{*}} \int_{0}^{y^{*}} q(y) d y+a_{k},
$$

where the sequence $a_{k} \in l^{2}$, i.e., $\left\|a_{k}\right\|=\left(\sum_{k=1}^{\infty} a_{k}^{2}\right)^{1 / 2}<\infty$. Equation (25) implies that the threshold value $y^{*}$ can be evaluated using

$$
y^{*}=\lim _{k \rightarrow \infty} \frac{k \pi}{\sqrt{\lambda_{k}}} \text {. }
$$

Thus, the domain size is reconstructed. The classical theories found in [3, 6, 10, 13, can then be directly applied to uniquely reconstruct the remaining, unknown part of $q(y)$. Moreover, efficient numerical algorithms have been developed for reconstructing $q(y)$ [5] 10, 25, and ultimately $\mu$ and $\sigma$ from the spectral data. However, the reconstruction of these spectral data from measurements is based on analytic continuation. This implies that the inverse problem is severely ill-posed [8] and that noise in the data is quite strongly amplified in the reconstruction; see section 7 Note that a similar approach was used in 23] in the context of the one-dimensional heat equation.

\section{Reconstruction of drift or diffusion term}

We showed in the preceding section that $q(y)$ and $y^{*}$ are uniquely determined from the measurements provided that one set of hypotheses stated in Theorems 1-3 (cf. Section 6) are verified. Combining equations (13) and (15), we find

$$
\nu^{\prime}(y)+\frac{\nu^{2}}{2}=2 q(y), \quad 0<y<y^{*} .
$$

Since both $\mu$ and $\sigma$ are known on $\left[0, x_{b}\right]$,

$$
\nu\left(y_{b}\right)=\left(\frac{\sqrt{2} \mu\left(x_{b}\right)}{\sigma\left(x_{b}\right)}-\frac{\sigma^{\prime}\left(x_{b}\right)}{\sqrt{2}}\right) .
$$

The Riccati equation (27), along with the boundary condition (28) admits a unique solution for $\nu(y)$.

Even if $\nu\left(0 \leq y \leq y^{*}\right)$ is known uniquely, one cannot extract both $\mu(x)$ and $\sigma(x)$ independently. However, if either $\mu$ or $\sigma$ is known, then the other one can uniquely be reconstructed from the knowledge of $\nu(y)$ on $\left(0, y^{*}\right)$. The simplest case occurs when only $\sigma$ is known, for then, so is $h(x)$, and the reconstruction of $\mu$ follows from the definition (11) of $\nu$. On the other hand, we can deduce from (91) and (11) that

$$
\left(\frac{\sigma^{\prime}}{2}-\frac{\mu}{\sigma}\right)(x)=-\frac{1}{\sqrt{2}} \nu\left(\int_{0}^{x} \frac{\sqrt{2}}{\sigma\left(x^{\prime}\right)} d x^{\prime}\right) .
$$

Let us assume that $\mu / \sigma$ can be expressed as a known (smooth) function of $\sigma$ and $x$. This includes the cases when $\mu$ is known or when $\mu / \sigma$ is known. Since $\sigma$ is $a$ 
priori known to be uniformly bounded from below, equation (29) provides a firstorder integro-differential equation that can then be solved for $\sigma(x)$ on $(0,1)$. The reconstruction is uniquely defined, concluding the proof of the main theorem.

The reconstruction of $q(y)$ on $y \in\left(0, y^{*}\right)$ clearly depends on the choice of $q(y)$ on $\left[0, y_{b}\right]$ since it would change the spectrum through Eq. (20). Therefore, a physically incorrect model of $q$ on $\left[0, y_{b}\right]$, contaminates the reconstruction of $\mu(x), \sigma(x)$ on $x \in(0,1)$, leading to physically incorrect, albeit unique, results. To be more specific, consider the simple case $\sigma^{2} \equiv 2$, whence $h(x)=x, y^{*}=1$, and $\nu(x)=\mu(x)$. Provided $|\mu|^{2} \ll\left|\mu^{\prime}\right|$, Eq. 15 implies $q(x) \sim \mu^{\prime}(x) / 2 \ll 1$. We then perform an asymptotic expansion of the eigenfunctions $\phi_{k}=\phi_{k 0}+\phi_{k 1}$ and eigenvalues $\lambda_{k}=\lambda_{k 0}+\lambda_{k 1}$, where $\phi_{k 1} \ll 1$ and $\lambda_{k 1} \ll 1$ (see [26] for instance), and obtain

$$
\lambda_{k 0}=\left(\pi\left(k-\frac{1}{2}\right)\right)^{2}, \quad \text { and } \quad \phi_{k 0}(x)=\cos \pi\left(k-\frac{1}{2}\right) x,
$$

and using the Fredholm alternative in the perturbation

$$
-\phi_{k 1}^{\prime \prime}+q \phi_{k 0}=\lambda_{k 1} \phi_{k 0}+\lambda_{k 0} \phi_{k 1}
$$

we obtain

$$
\lambda_{k 1}=\frac{\int_{0}^{1} q(x) \phi_{k 0}^{2}(x) d x}{\int_{0}^{1} \phi_{k 0}^{2}(x) d x} .
$$

Using the relation $\cos 2 \theta=2 \cos ^{2} \theta-1$, we deduce from the above equation that the knowledge of $\lambda_{k 1}$ allows us to obtain

$$
q_{2 m-1}=\frac{1}{2} \int_{0}^{1} q(x) \cos (2 m-1) \pi x d x .
$$

Therefore the knowledge of one spectrum $\lambda_{k}$ gives us half of the cosine Fourier transform of $q(x)$, hence of $\mu(x)$. The other half needs to be known, for instance by imposing that we know $q(x)$, or equivalently $\mu(x)$, on $(0,1 / 2)$.

Although we have shown what parts of $\mu(x)$ and $\sigma(x)$ can be uniquely reconstructed from a single first-exit time distribution, in practice, finding the eigenvalues $\lambda_{k}$ from data to the precision required for accurate reconstruction is difficult.

\section{Reconstruction with multiple measurements}

We have seen in the preceding section that either $\mu$ or $\sigma$ could be reconstructed from the knowledge of $q$ provided the other one is known. In this section we obtain sufficient conditions on the measurements so that $q(x)$ can uniquely be reconstructed on $(\varepsilon, 1)$ for $\varepsilon>0$. We show that it is sufficient to measure $u\left(y_{m} ; \lambda\right)$ at a countable sequence of points $y_{m}$ with accumulation point at $y=0$. These points $y_{m}$ correspond to points $x_{m}$ via the transform $x_{m}=h^{-1}\left(y_{m}\right)$. They are constructed as follows.

We choose $y_{0}$ such that $y_{0} \geq y^{*} / 2$ and then by induction the points $y_{m}(m \geq 1)$ such that $y_{m} \in\left(y_{m-1} / 2, y_{m-1}\right)$. We assume that $y_{m} \rightarrow 0$ as $m \rightarrow \infty$. We denote by $\mathcal{Y}$ the set of sequences $\left\{y_{m}\right\}_{m \in \mathbb{N}}=\left(y_{0}, y_{1}, \cdots\right)$ satisfying the above constraints. We can then show the following result 
Theorem 3 Let us assume that $q \in L^{2}(0,1)$, that there is a small interval $[0, \varepsilon]$ where $q(y)$ is constant and that there is a known index $M$ such that $y_{M}<\varepsilon$. The parameters $\varepsilon$ and $y_{M}$ need not be known. Then the measurements at $u\left(y_{m} ; \lambda\right), 1 \leq m \leq M+1$, for $\left\{y_{m}\right\} \in \mathcal{Y}$ and $\lambda>0$ uniquely determine $q(y)$ on $\left(0, y^{*}\right)$ as well as the point $\left\{y_{m}\right\}_{m \in \mathbb{N}}$ and $y^{*}$.

Proof. By hypothesis, we know $M$ such that $y_{M}<\varepsilon$. Consider the value of $u\left(y_{M} ; \lambda\right) \neq 0$. If $u\left(y_{M} ; \lambda\right)=0$, the condition at $y=0$ would imply that $u(y ; \lambda) \equiv 0$ on $\left(0, y_{M}\right)$ and, hence on $\left(y_{M}, y^{*}\right)$, since then $u\left(y_{M} ; \lambda\right)=\frac{\partial u}{\partial y}\left(y_{M} ; \lambda\right)=0$. This however contradicts the fact that $u\left(y^{*}\right)=1$. Upon dividing the solution $u(y ; \lambda)$ by $u\left(y_{M} ; \lambda\right) \neq 0$ we deduce that $v_{M}(y ; \lambda)=u(y ; \lambda) / u\left(y_{M} ; \lambda\right)$ solves

$$
\begin{aligned}
& v_{M}^{\prime \prime}(y ; \lambda)-q(y) v_{M}(y ; \lambda)=\lambda v_{M}(y ; \lambda), \quad 0<y<y_{M} \\
& v_{M}\left(y_{M} ; \lambda\right)=1, \quad \frac{\partial v_{M}(0 ; \lambda)}{\partial y}=0 .
\end{aligned}
$$

We then know from the proof of Theorem 2 and the asymptotic expansion (25) that the measurement of $v\left(y_{M+1}\right)$ uniquely determines $y_{M}$ and $q(y)$ on $\left(0, y_{M}\right)$ since the latter is constant.

The rest of the proof follows by induction. Let us assume that $q(y)$ is known on $\left(0, y_{m+1}\right)$ for $m+1 \leq M$. Then $u\left(y_{m} ; \lambda\right) \neq 0$ and $v_{m}(y ; \lambda)=u(y ; \lambda) / u\left(y_{m} ; \lambda\right)$ satisfies the same equation (30) with $y_{M}$ replaced by $y_{m}$. Upon using Theorem 2 we deduce that the knowledge of $v_{m}\left(y_{m+1} ; \lambda\right)$ for $\lambda>0$ uniquely determines $y_{m}$ and $q(x)$ on $\left(0, y_{m}\right)$. This allows us to reconstruct $y_{0}$ and $q(y)$ on $\left(0, y_{0}\right)$ by induction. Applying Theorem 2 on $\left(0, y^{*}\right)$ one last time concludes the proof of the theorem.

The same type of proof could be used with other local a priori information on the behavior of $q(y)$ in the vicinity of $y=0$. For instance, we can show that the reconstruction is feasible provided that $q(x)$ is known on an arbitrarily small interval $(0, \varepsilon)$ and provided that there exists an admissible sequence $\left(y_{0}, y_{1}, \cdots, y_{M}\right)$ of measurements with $y_{M} \leq \varepsilon$.

In both cases the reconstruction of $q(y)$ and of the measurement points $y_{m}$ allows us to obtain some information about the original drift and diffusion terms $\mu(x)$ and $\sigma(x)$. We can for instance prove the following result.

Theorem 4 Assume that $\mu(x)$ and $\sigma(x)$ are known on $(0, \eta)$ for some $\eta>0$ and that $w\left(x_{m} ; \lambda\right)$ is measured for $\lambda>0$ at $x_{m}, 1 \leq m \leq M$, where the constraints on $\left\{x_{m}\right\}_{m}$ are that $x_{M} \leq \eta$ and $\left\{y_{m}\right\}_{m}=\left\{h\left(x_{m}\right)\right\}_{m} \in \mathcal{Y}$. If, in addition, either $\mu(x), \sigma(x)$, or $\mu(x) / \sigma(x)$ is known on $[0,1]$, then both $\mu(x)$ and $\sigma(x)$ are uniquely determined on $[0,1]$.

Proof. The proof follows by induction. We define $y_{M}=h\left(x_{M}\right) \leq \varepsilon=h(\eta)$. Let us now define $f_{M}(y)$ as in (13) on $\left(0, y_{M-1}\right)$ but with boundary condition $f_{M}\left(y_{M}\right)=1$. This implies that $u_{M}\left(y_{M} ; \lambda\right)=w\left(x_{M} ; \lambda\right)$ where $u_{M}(y ; \lambda)$ solves the Schrödinger equation

$$
\begin{aligned}
& u_{M}^{\prime \prime}(y)-q(y) u_{M}(y)=\lambda u_{M}(y), \quad 0<y<y_{M-1} \\
& u_{M}\left(y_{M-1}\right)=\frac{1}{f\left(y_{M-1}\right)}>0, \quad \frac{\partial u_{M}(0 ; \lambda)}{\partial y}=0 .
\end{aligned}
$$

We have seen in Theorem 3 that $q(y)$ can then be uniquely reconstructed on $\left(0, y_{M-1}\right)$ since it is known on $\left(0, y_{M}\right)$. We can now apply the rest of the proof of Theorem 2 on the interval $\left(0, x_{M-1}\right)$. This allows us to reconstruct $\mu$ and $\sigma$ uniquely on $\left(0, x_{M-1}\right)$. 
Similarly the knowledge of $\mu$ and $\sigma$ on $\left(0, x_{m}\right)$ allows us to reconstruct both coefficients uniquely on $\left(0, x_{m-1}\right)$ and the same knowledge on $\left(0, x_{0}\right)$ allows reconstruction on $(0,1)$. This concludes the proof of the theorem.

\section{Implementation of the reconstruction}

Theorems 10 2 and 4provide an explicit method to reconstruct the drift and diffusion coefficients $\mu$ and $\sigma$ from the measurements. This method is based on three steps. The first step consists of finding the poles of meromorphic functions of the form $\lambda \rightarrow u(y ; \lambda)$ at fixed $y$ from its knowledge for $\lambda>0$. In the second step, the potential $q(y)$ is reconstructed from the knowledge of the spectral data provided in step 1. Finally $\mu(x)$ and $\sigma(x)$ are reconstructed from $q(y)$ by solving first-order ordinary differential equations.

Step 3 is well-posed in the sense that noise in the potential $q(y)$ are at most linearly amplified in the reconstruction of $\mu(x)$ and $\sigma(x)$. Step 2 is also well posed as the reconstruction of the potential $q(y)$ from the spectra is stable, for instance in the sense that an error of order $\varepsilon$ on the $l^{2}$ norm of $a_{k}$ in (25) implies an error also of order $\varepsilon$ in the reconstruction of $q(x)$ in the $L^{2}$ sense 19,25 .

Step 1, however, is severely ill-posed in the sense that order- $\varepsilon$ errors in the measured generate errors greater than order $\varepsilon^{\alpha}$ (for all $\alpha>0$ ) in the $l^{2}$ norm of the coefficients $a_{k}$ in (25). This is a similar result to the reconstruction of diffusion coefficients from boundary measurements, which is known to be severely ill-posed [1] 13. We can show a Hölder-type stability result in the reconstruction of each eigenvalue. This means that for each eigenvalue $\lambda_{k}$, there exists $\alpha_{k}>0$ such that errors in the data of order $\varepsilon$ yield an error of order $\varepsilon^{\alpha_{k}}$ on the reconstruction. Unfortunately, the prefactor in front of $\varepsilon^{\alpha_{k}}$ grows exponentially in $k$ so that at most only approximately $|\log \varepsilon|$ eigenvalues can be reconstructed from oder- $\varepsilon$ accurate measured data. This behavior is a consequence of the results on the meromorphic prolongation of functions with poles, as shown by K. Miller [21]. Since we do not attack the numerical reconstruction here we briefly sketch the result and how it may be used to derive the above stability estimates.

Let us consider the open disk $D$ of radius $R$ and center 0 in the complex plane and a meromorphic function $f(\lambda)=u\left(y_{0} ; \lambda\right)$ inside that disk with $N$ poles, which we assume lie on the segment $(-R, 0)$. We want to reconstruct these $N$ poles from the measurement of the meromorphic function on the segment $\Gamma=(0, R)$. Let us define by $w(z)$ the function that takes the value 0 on $\partial D$, the value 1 on $\Gamma$, and is harmonic on $D \backslash \Gamma$. One verifies that $0<w(z)<1$ on $D \backslash \Gamma$. Let us recast our meromorphic function as $f(\lambda)=F(\lambda) / \prod_{k=1}^{N}\left(\lambda-\lambda_{k}\right)$ where $F(\lambda)$ is analytic on $D$. We assume that

$$
|F-B h|<\varepsilon|B| \quad \text { on } \quad \Gamma, \quad \text { and } \quad|F|<E, \quad \text { on } \quad \partial D .
$$

Here $h(\lambda)$ are our measurements. The above relation indicates what we mean by error in the measurements and essentially says that $f-h$ is bounded by $\varepsilon$ in the supremum norm. Upon slightly modifying the proof of Theorem 1 in [21], we obtain that it is possible to reconstruct the poles $\lambda_{k}$ of $f$ on $D$ with an error of order

$$
\left|\delta \lambda_{k}\right| \leq \frac{C^{N} R^{N}\left(\varepsilon R^{N}\right)^{w\left(\lambda_{k}\right)} E^{1-w\left(\lambda_{k}\right)}}{\left|W_{k}\right|\left(\prod_{l \neq k}\left|\lambda_{l}-\lambda_{k}\right|\right)^{2}} .
$$


Here we have bounded $B$ by $R^{N}$ on $D$. In the case of interest in this paper, we have that

$$
R \sim N^{2}, \quad \lambda_{k} \sim k^{2}, \quad \text { and } \quad W_{k}=u\left(y^{*}\right) \phi_{k}\left(y_{0}\right) \phi_{k}^{\prime}\left(y^{*}\right) .
$$

The radius is chosen as roughly $1 / 2\left(\lambda_{N}+\lambda_{N+1}\right)$. At the boundary $\partial D$ of the domain, we observe that $F$ is bounded by (using Stirling's approximation)

$$
E \sim \prod_{l \neq k}\left|\lambda_{l}-\lambda_{k}\right| \sim(N !)^{2} \approx N^{2 N+1} e^{-N} .
$$

Using these values we find that

$$
\left|\delta \lambda_{k}\right| \leq C^{N} \varepsilon^{w\left(\lambda_{k}\right)}
$$

for some positive constant $C$. The above constraint is sharp [21]. This shows that the reconstruction of any eigenvalue is Hölder continuous, where the order of continuity $\alpha=w\left(\lambda_{k}\right)$. This is therefore a mildly ill-posed problem.

However, although the reconstruction of each eigenvalue is mildly ill-posed, collectively the reconstruction of the whole spectrum is severely ill-posed if one wants an approximation in the $l^{2}$ sense. Indeed for a given noise level $\varepsilon$, the above formula shows that we can only reconstruct a number of eigenvalues that is proportional to $|\log \varepsilon|$, for the reconstructed information becomes useless as $\left|\delta \lambda_{k}\right| \geq 1$ and the corresponding coefficients $a_{k}$ in (25) are not reconstructed at all. Now assuming that $w\left(\lambda_{k}\right)=1$, which is the best we can expect, we observe that $C^{N} \varepsilon=1$ for $N \sim|\ln \varepsilon|$. This shows that the reconstruction of the potential $q(y)$ is severely ill-posed. Indeed let us assume that the asymptotic coefficient of $q$ in (25) are of the form $a_{k} \sim k^{-M}$ for some $M$. The $l^{2}$ error on the Fourier coefficients obtained by truncating all coefficients of index greater than $|\ln \varepsilon|$ is then of order $N^{-M+1 / 2} \sim|\log \varepsilon|^{-M+1 / 2} \gg \varepsilon^{\alpha}$ for all $\alpha>0$.

\section{Summary and Conclusions}

We have shown that if $\mu$ and $\sigma$ are known in say, $x \in\left[0, x_{b}\right]$, with $x_{b}=h^{-1}\left(y^{*} / 2\right)$ (Eq. [18), one of them can be reconstructed from a single first-exit time distribution measurement at $x_{0} \notin \Lambda$. The procedure is formally carried out by transforming the backward Kolmogorov equation for the first-exit time distribution $w(x ; \lambda)$ into a Schrödinger equation in $u(y ; \lambda)$ with a potential $q(y)$ that is a functional of $\mu(x)$ and $\sigma(x)$. If this potential is known in the domain $0 \leq y \leq y_{b} \equiv h\left(x_{b}\right), q(y)$ can be reconstructed from the single eigenvalue spectrum obtained by analytically continuing the solution $u(y ; \lambda)$ (in $\lambda$ ) and finding the poles. It is this pole-finding procedure that is severely ill-posed. The asymptotic limit of the eigenvalues also determine the extent of the transformed domain $y^{*}$. From the reconstructed $q(y)$, one can reconstruct $\mu(x)$ or $\sigma(x)$, only if the other (or $\mu(x) / \sigma(x)$ ) is also known. Finally, we have shown that the measurement process and the determination of spectra can be repeated a sufficient number of times to obtain $q(y)$ (Theorem 3) and $\mu$ or $\sigma$ (Theorem 4) on the entire domain provided $q(y)$ and $\sigma$ or $\mu$ is initially known on a small interval $(0, \varepsilon)$.

Our analysis in section 7 shows that the proposed inversion is severely ill-posed even if the hypotheses of the aforementioned theorems are satisfied. However the reconstruction of partial spectral data is mildly ill-posed so we believe the proposed method can be implemented. Let us conclude with the following remark. Our method is based on reconstructing the eigenvalues $\lambda_{k}$ from the knowledge of $u\left(x_{0} ; \lambda\right)$. The 
latter may however provide additional information. For instance we observe from (21) that the residuals $\phi_{k}(y) \phi_{k}^{\prime}\left(y^{*}\right) u\left(y^{*}\right)$ can also be reconstructed from the measured data. It is known that the measurement of one spectrum $\left\{\lambda_{k}\right\}$ may be sufficient to uniquely reconstruct $q(y)$ on the whole interval $\left(0, y^{*}\right)$ provided that additional normalization conditions be also measured on the eigenfunctions [18, 25]. It is not clear whether additional information on $\phi_{k}(y) \phi_{k}^{\prime}\left(y^{*}\right) u\left(y^{*}\right)$ may be useful. Preliminary numerical simulations seem to show that reconstructions from a single measurement only allows us to reconstruct part of the potential.

\section{Acknowledgments}

This work was supported by the National Science Foundation (US) through grants DMS-0239097 (GB) and DMS-0206733 (TC) and by the Office of Naval Research through grant N00014-02-1-0089 (GB). GB also acknowledges support from an Alfred P. Sloan Fellowship.

\section{References}

[1]Alessandrini G 1990 Singular solutions of elliptic equations and the determination of conductivity by boundary measurements J. Differ. Equ. $84252-273$

[2]Bender C M and Orszag S A 1999 Advanced Mathematical Methods for Scientists and Engineers: Asymptotic Methods and Perturbation Theory (New York: Springer-Verlag)

[3]Borg G 1946 Eine Umkehrung der Sturm-Liouville Eigenwertaufgabe Acta Math. 76 1-96

[4]Breiman L 1992 Probability, Classics in Applied Mathematics (Philadelphia, PA: SIAM)

[5]Brown B M, Samko V S, Knowles I W, and Marletta M 2003 Inverse spectral problem for the Sturm-Liouville equation Inverse Problems 19 235-252

[6]Chadan K, Colton D, Päivärinta L, and Rundell W 1997 An Introduction to Inverse Scattering and Inverse Spectral Problems (Philadelphia, PA: SIAM)

[7]Doering C R and Gadoua J C 1992 Resonant activation over a fluctuating barrier Phys. Rev. Lett. 69 2318-2321

[8]Engl H W, Hanke M and Neubauer A, 1996 Regularization of Inverse Problems (Kluwer Academic Publishers, Dordrecht)

[9]Gardiner C W 2002 Handbook of Stochastic Methods for Physics, Chemistry and the Natural Sciences $2^{\text {nd }}$ ed. (Berlin: Springer)

[10]Gelfand I M, and Levitan B M 1955 On the determination of a differential equation from its spectral function Am. Math. Soc. Transl. 1 253-304

[11]Gerstner W and Kistler W M 2002 Spiking neuron models: single neurons, population, and plasticity (Cambridge: Cambridge University Press)

[12]Gikhman I I and Skorokhod A V 1972 Stochastic Differential Equations (New York: SpringerVerlag)

[13]Hochstadt H and Lieberman B 1976 An inverse Sturm-Liouville problem with mixed given data SIAM J. Appl. Math. 34 676-680

[13]Isakov V 1998 Inverse Problems for Partial Differential Equations (New York: Springer-Verlag)

[14]Johnston D and Wu S M S 1995 Foundations of Cellular Neurophysiology (Cambridge, MA: MIT Press)

[15]van Kampen N G 1981 Ito versus Stratonovich J. Stat. Phys. 24 175-187

[16]Kramers H A 1940 Brownian motion in a field of force and the diffusion model of chemical reactions Physica 7 284-304

[17]Lange K L 2003 Applied Probability (New York: Springer-Verlag)

[18] McLaughlin J R 1986 Analytical methods for recovering coefficients in differential equations from spectral data SIAM Rev. 28(1) 53-72

[19]McLaughlin J R 1988 Stability theorems for two inverse spectral problems Inverse Problems. 4(2) 529-540

[20]Merkel R, Nassoy P, Leung A, Ricthie K, and Evans E 1999 Energy landscapes of receptor-ligand bonds explored with dynamic force spectroscopy Nature 397 50-53

[21]Miller, K 1970 Stabilized Numerical Analytic Prolongation with Poles S.I.A.M. J. Appl. Math. 18(2) 346-363 
[21]Nykamp D Q Measuring linear and quadratic contributions to neuronal response, Submitted 2003 [22] Øksendal B Stochastic Differential Equations (Berlin: Springer-Verlag)

[23]Ramm A G 2001 An inverse problem for the heat equation, J. Math. Anal. Appl. 264 691-697

[24]Risken H 1996 The Fokker-Planck Equation, $2^{\text {nd }}$ ed. (New York: Springer)

[25]Rundell W, and Sacks P E 1992 Reconstruction techniques for classical inverse Sturm-Liouville problems Math. Comput. 58 161-183

[26]Rundell W, and Sacks P E 1992 The reconstruction of Sturm-Liouville Operators Inverse Problems 8 457-482

[27]Seifert U 2000 Rupture of Multiple Parallel Molecular Bonds under Dynamic Loading Phys. Rev. Lett. 84 2750-2753

[28] Tuckwell H C 1976 On the First-exit Time Problem for Temporally Homogeneous Markov Processes J. Appl. Prob. 13 39-48

[29] Tuckwell HC and Wolfgang R 1978 Neuronal Interspike Time Distributions and the Estimation of Neurophysiological and Neuroanatomical Parameters J. theor. Biol. 71 167-183

[30]Tuckwell H C and Cope D K 1980 Accuracy of Neuronal Interspike Time Calculated from a Diffusion Approximation J. theor. Biol. 83 377-387

[31]Tuckwell H C 1989 Stochastic Processes in the Neurosciences (Philadelphia, PA: SIAM) 